\title{
El Deterioro Cognitivo como una complicación de la Diabetes Mellitus Tipo 2
}

\author{
Congnitve Impairtent as a Type 2 Diabestes Mellitus complication
}

Shadye Rocío Matar-Khalil ${ }^{1}$, Fabio Camilo Rubio-Sandoval ${ }^{2}$

\section{Resumen}

Introducción. La Diabetes Mellitus es una enfermedad compleja que impacta la salud pública debido a su alta prevalencia, el costo que implica y complicaciones asociadas como lo es la alteración cognitiva. Metodología. Se realizó una revisión de tema consultando literatura nacional e internacional en bases de datos de ciencias sociales y ciencias de la salud entre los ańos 1990-2019 que exponen la posible asociación entre la diabetes mellitus y deterioro cognitivo, así como las posibles rutas que lo conllevan. Resultados. La literatura consultada plantea una relación entre la diabetes mellitus y complicaciones del sistema nervioso central, lesiones neurológicas, deficiencias en la capacidad de atención, pérdida de la memoria, deterioro cognitivo, un considerable incremento en el riesgo de desarrollar demencia y demencia tipo Alzheimer, sin embargo, no se evidencia un consenso en el grado de afectación que pueda tener sobre los procesos cognitivos. Conclusiones. La naturaleza progresiva tanto de la diabetes mellitus, así como el riesgo vascular asociado, sugieren la necesidad de realizar prevención del deterioro cognitivo en pacientes diagnosticados con diabetes mellitus, a partir de una evaluación integral que incluya pruebas neuropsicológicas dentro de los exámenes de rutina.

Palabras claves: cognición, diabetes mellitus, complicaciones de la diabetes. 


\section{Abstract}

Introduction: Diabetes mellitus is a complex and chronic disease that has an impact on public health due to its high prevalence, the implicit economic cost and associated complications like cognitive alterations. Methodology: A review of the topic was carried out across national and international literature in databases of social sciences and health sciences between the years 1990-2019 that expose the possible association between diabetes mellitus and cognitive impairment, as well as the possible routes that lead to cognitive alterations. Results: The consulted literature exposed a link between diabetes mellitus and complications of the central nervous system, neurological lesions, attention capacity deficiencies, memory loss, cognitive impairment, and a considerable increase in the risk of developing dementia and Alzheimer's type dementia. Conclusions: The progressive nature of both diabetes mellitus, and associated vascular risk, suggest the need for prevention of cognitive impairment based on a comprehensive evaluation that includes neuropsychological tests within routine examinations in patients diagnosed with diabetes mellitus.

Keywords: cognition, diabetes mellitus, diabetes complications.

\section{Introducción}

De acuerdo con estimaciones realizadas por la Federación Internacional de Diabetes, para el año 2019 se contaban 463 millones de adultos (20-79 ańos) con diabetes mellitus (DM) en todo el mundo y se calcula que este número aumentará a 578.4 millones para el año 2030 y 700.2 millones para el año 2045 (1). Existe un marcado incremento en la prevalencia de la enfermedad desde mitad del siglo pasado (2), tendencia que se mantendrá durante el presente siglo a medida que las condiciones y estilos vida de las poblaciones humanas se tornen cada vez más sedentarias, y con mayor velocidad de aumento en países en vías de desarrollo (1-3).
La mayoría de los nuevos casos de diabetes mellitus tipo 2 (DM2) ocurren en un contexto de estilo de vida sedentario; dietas ricas en grasas y poco ejercicio físico, lo que conduce al aumento de la obesidad, resistencia a la insulina, hiperinsulinemia compensadora, insuficiencia de células $\beta \mathrm{y}$, finalmente DM (4).

Además, se calcula que 300 millones de personas presentan características que indican un riesgo de sufrir DM2 en el futuro, como hiperglicemia en ayunas, intolerancia a la glucosa (IG), diabetes mellitus gestacional (DMG) y resistencia a la insulina (RI) en euglucemia (4). Lo cual hace que desde un punto de vista epidemiológico la diabetes 
sea una de las enfermedades crónicas no transmisibles con mayor importancia para los programas mundiales de salud, e implica un mayor número de personas que deberán ser atendidas a causa de complicaciones relacionadas a dicho síndrome metabólico durante los próximos años.

La diabetes tipo 1 (DM1) ha sido típicamente relacionada con pacientes jóvenes, mientras que la DM2 se ha relacionado exclusivamente a pacientes adultos, sin embargo, de acuerdo con la Organización Mundial de la Salud (OMS) el nùmero de pacientes jóvenes diagnosticados con DM2 se encuentra en aumento (3) posiblemente debido al incremento en las tasas de sobrepeso y obesidad en este grupo poblacional, condición descrita como un factor de riesgo en el desarrollo de la patología (4)

A medida que aumenta el número de pacientes diagnosticados con DM2 también es posible encontrar un mayor numero de publicaciones que plantean una relación entre la diabetes y complicaciones del sistema nervioso central, lesiones neurológicas, deficiencias en la capacidad de atención, pérdida de la memoria, y deterioro cognitivo; aunque en la actualidad no se ha logrado un consenso que establezca el nivel de asociación entre estas variables y la presencia de DM2. Por tanto, este articulo presenta una revisión de publicaciones durante los últimos veinte años que exponen la posible asociación entre la DM2 y deterioro cognitivo, así como posibles rutas que conllevan al deterioro de la cognición.

\section{Metodología}

Se hizo una revisión de tema consultando literatura nacional e internacional de los últimos veinte años en las bases de datos de ciencias sociales y ciencias de la salud (Web of Science, PsycoInfo, PubMed, Dialnet, Redalyc, ScieELO, ScienceDirect Medline) en ingles y español, usando como descriptores para la búsqueda diabetes mellitus tipo 2, funciones cognitivas, deterioro cognitivo y demencia. Los artículos fueron seleccionados teniendo como criterio que el objetivo del estudio abordara la evaluación neuropsicologica a pacientes diabéticos tipo 2 y estudios que explicaran la posible asociación entre la DM2 y el deterioro cognitivo, así como posibles rutas que conllevan al deterioro de la cognición.

\section{Diabetes}

La diabetes es descrita como "un grupo de enfermedades metabólicas caracterizadas por la presencia de hiperglicemia crónica (aumento anormal de la cantidad de glucosa en sangre), con alteración del metabolismo de los carbohidratos, grasas y proteinas... debido a dos defectos metabólicos implicados en la etiologia de la enfermedad; La resistencia a la insulina o el déficit en su secreción (5)".

El término metabolismo describe los procesos de interconversión de compuestos químicos en el organismo, las vías que siguen 
las moléculas individuales y sus interrelaciones. Una de las moléculas de mayor importancia para el adecuado funcionamiento del metabolismo es la glucosa toda vez que este carbohidrato es el principal combustible metabólico de casi todos los tejidos, en especial para el sistema nervioso central y eritrocitos (6).

A diferencia de las células hepáticas que son permeables a la glucosa, es decir que permiten su paso libremente, las células extrahepáticas son relativamente impermeables a la glucosa, y su transporte a través de las membranas celulares está mediado por la hormona insulina, producida y secretada por células $\beta$ de los islotes de Langerhans, en el páncreas, en respuesta a hiperglucemia, estimulando al hígado para que almacene glucosa como glucógeno, y facilitando la captación de glucosa hacia tejidos extrahepáticos (6).

No obstante, el metabolismo de carbohidratos y por ende el control de los niveles de glucosa en sangre se ven alterados de dos formas particulares, a través de la incapacidad del individuo para sintetizar y secretar insulina debido a la progresiva destrucción de las células $\beta$ pancreáticas como ocurre en la DM1, y a través de la combinación entre la resistencia a la acción de la insulina (sensibilidad alterada) por parte de los tejidos y una inadecuada respuesta en la compensación de la euglicemia como ocurre en la DM2 (7).

La destrucción de las células $\beta$ pancreáticas, en la DM1, se debe a una reacción au- toinmune, en la que el sistema de defensa del cuerpo ataca y destruye a dichas células. Como resultado, el páncreas deja de producir la insulina necesaria para la absorción de azúcar presente en la sangre (4). Por lo tanto, las personas que padecen DM1 necesitan la administración de frecuentes dosis de insulina (insulino dependientes) con el fin decontrolar sus niveles de azúcar y mantener un adecuado metabolismo de los carbohidratos ingeridos.

En la DM2 aun cuando el páncreas si puede producir insulina los tejidos presentan una sensibilidad alterada a su acción, de modo que la insulina producida se torna ineficaz en el proceso de asimilación de la glucosa (insulino requiriente), lo cual se traduce en episodios recurrentes de hiperglicemia, y aun cuando la etiología de la DM2 se encuentra en estudio, existen varios factores de riesgo que cobran una significativa relevancia en su desarrollo; el sobrepeso, la inactividad física o sedentarismo y una nutrición pobre.

La diabetes al involucrar procesos metabólicos, es la causa de una gran variedad de complicaciones que involucran a todos los sistemas del organismo y entre las cuales se encuentran; retinopatìa, nefropatìa, neuropatias asociadas a múltiples manifestaciones, disfunción cognitiva, diversas manifestaciones de enfermedad vascular, enfermedad coronaria, enfermedad vascular periférica, alteraciones metabólicas asociadas con la dislipidemia (Niveles anormales de lípidos en la sangre) cetoacidosis e hipoglicemia (3, $5,7)$. 
Las complicaciones de la diabetes han sido divididas en dos grupos; complicaciones microvasculares (microangiopatía) y complicaciones macrovasculares, las primeras se refieren a aquellas complicaciones relacionadas con afectaciones de capilares, mientras las segundas hacen referencia a afectaciones de grandes vasos sanguíneos. Dentro de las complicaciones microvasculares se incluyen la retinopatía (afectación de la retina) que representa la primer causa de ceguera irreversible en personas en edades de 16 a 64 en países en vías de desarrollo (8) la nefropatía (afectación de glomérulos renales) que representa una elevada carga de morbimortalidad al evolucionar a insuficiencia renal y neuropatía (afectación de nervios periféricos) que puede evolucionar en ulceración, infección y amputación de extremidades inferiores debido al dańo en los nervios acompañado de alteracion en la circulación en los pies como resultado de daño en vasos sanguíneos (4). Las complicaciones macrovasculares incluyen enfermedad coronaria que es la causa de muerte más común entre los pacientes diabéticos, trombosis e hipertensión arterial $(5,9)$.

De manera particular las afectaciones al sistema nervioso central relacionadas a la presencia de diabetes han sido reportadas desde inicios del siglo XX. En la década de 1920 Miles y Root (10) ya planteaban una relación entre la diabetes y lesiones neurológicas, psicosis, deficiencias en la capacidad de atención y pérdida de la memoria; posteriormente Dejgaard estimaba que la prevalencia de afectación en el sistema ner- vioso central o "encefalopatía diabética" en pacientes con diabetes podría ser superior al 40\% (11), más recientemente se ha asociado a la diabetes con un bajo desempeño cognitivo y un incremento en el riesgo de desarrollar demencia (12-15). Dichas complicaciones parecen estar vinculadas al mantenimiento de altos niveles de glicemia a través del tiempo en pacientes diagnosticados con diabetes (16), toda vez que las condiciones fisiológicas generadas por un estado crónico de hiperglicemia conllevan un exceso oxidativo de moléculas de glucosa y la glicación no enzimática de proteínas, causando daño estructural en tejidos y órganos $(17,18)$.

Una condición crónica de hiperglucemia ya sea por la baja producción de insulina en las células pancreáticas o por la resistencia a su acción y en consecuencia fallas en la asimilación de glucosa, genera el desabastecimiento del combustible metabólico necesario para el funcionamiento celular, incluido la generación de potenciales de acción en axones neuronales, así como también ocasiona la glicación no enzimática de proteínas, factor que actúa como un elemento neurodegenerativo (19-22) y que podría contribuir en la aparición de anormalidades micro-estructurales de la materia blanca cerebral $(23,24)$, o anomalías de origen vascular debidas a infartos y micro infartos de la vasculatura cerebral relacionados a procesos de demencia en pacientes con DM2 (25).

Por lo tanto, y partiendo de la premisa que no se ha desarrollado un tratamiento 
que permita la cura de la diabetes, la prevención de las complicaciones relacionadas, tanto en pacientes diagnosticados con DM1 como DM2 ha centrado su atención en el monitoreo y control de los niveles de la glicemia a través de la evaluación periódica de los niveles de hemoglobina glicosilada (HbA1c), con el objetivo de mantener los niveles de azúcar en un punto en el cual se disminuya el riesgo de la presentación de complicaciones micro o macro vasculares (26-28).

\section{Cognición y diabetes}

La cognición puede ser entendida como el proceso del pensamiento, o como el procesamiento mental de la información (29). La cognición incluye aquellos procesos a través de los cuales un estímulo interno o externo, es captado mediante la percepción, es transformado en seńales comprensibles por el cerebro, es reducido a aquellos elementos que ameritan atención, es elaborado y almacenado en la memoria, recuperado y usado a través no solo de pensamientos sino también a través de las acciones resultado de los estímulos (30). De tal forma que la mente es un activo selector de información del ambiente, relacionando dicha información con el conocimiento previamente adquirido y generando una respuesta sobre los resultados del proceso.

Por lo tanto, el individuo no actúa como una entidad pasiva que se encuentra a mer- ced de las señales del entorno. Por el contrario, el individuo a través de la cognición tiene la capacidad de buscar información enfocando de manera consciente sus recursos atencionales, seleccionarla, manipularla $y$ reconstruirla en forma de memorias, en un proceso que ocurre bajo un esquema de interacción entre la información proveniente del entorno, la naturaleza del sistema de procesamiento y los conocimientos previos (31).

Lo que el individuo conoce acerca del entorno ha sido intervenido por la actividad cognitiva en la medida en que la cognición brinda el ensamblaje necesario para interpretar y reinterpretar la información sensorial, relativa a las características propias de los elementos del entorno, que es captada por los órganos de los sentidos. Interactuando en esta intervención aspectos de la cognición como las sensaciones, percepciones, imaginación, retención, recuperación, resolución de problemas y pensamiento (32).

Así, en los procesos cognitivos intervienen múltiples funciones mentales superiores, mencionadas por Ulric Neisser como "hipotéticas etapas o aspectos de la cognición" (31) y que en un contexto cotidiano pueden ser entendidas como habilidades; el lenguaje, la imaginación, la planificación, el control ejecutivo de las acciones, la percepción, atención, memoria, toma de decisiones, razonamiento y resolución de problemas. En consecuencia, estas funciones, habilidades, juegan un importante papel en el desenvolvimiento e interacción del individuo 
en y con el entorno, en la percepción de sí mismo y la percepción de otros individuos.

Los procesos cognitivos son susceptibles a cambios que se presenten en su sustrato neurológico, por lo tanto, procesos bioquímicos adversos que se den de forma sostenida por largos periodos de tiempo como ocurre a los pacientes con DM2 y que conlleven algún tipo de daño o cambios a nivel neural, tendrán repercusión en los procesos cognitivos. De tal forma que el procesamiento mental de la información puede verse comprometido toda vez que surgen cambios en áreas del cerebro asociadas a uno o más dominios cognitivos; funciones ejecutivas, velocidad de procesamiento, atención y memoria entre otros, y aun cuando estos cambios se dan de manera natural y continua, gracias a la plasticidad cerebral, también conocida como neuroplasticidad, que favorece la adaptación del cerebro durante el desarrollo del individuo, se pueden generar consecuencias negativas al presentarse de manera sostenida y prolongada bajo situaciones de estrés metabólico, $(19,20)$, al igual que del estrés psicológico tal como parece ocurrir debido a las variaciones tanto de insulina como de glucosa a nivel de cerebro en los pacientes con diabetes (21). Al respecto, Strachan plantea una asociación entre la hiperglicemia aguda y deficiencias en la memoria de trabajo y atención (33), en contraste, un temprano control de la hiperglicemia tiene el potencial para reducir el riesgo de complicaciones micro y macro vasculares (17).
Sobre la asociación entre DM1 y deficiencias cognitivas, la literatura describe afectaciones en múltiples dominios cognitivos (11, 34-36), sin embargo, estas manifestaciones han sido descritas en menor frecuencia y magnitud en pacientes con DM1 en comparación a pacientes con DM2 (11, 37). Adicionalmente, a través de imágenes de resonancia magnética se han detectado anormalidades micorestructurales de la materia blanca relacionadas a la presencia de diabetes $(23,24)$ pero significativamente más profundas en pacientes diagnosticados con DM2 en comparación con pacientes diagnosticados con DM1 (23) y las cuales se han asociado con lentitud en la velocidad de procesamiento de la información y un bajo desempeño en el dominio memoria (24). Por su parte Jacobson et al. (38) reportan no encontrar evidencia sustancial de un declive en la función cognitiva al realizar un estudio longitudinal por un periodo de 18 ańos en 1144 pacientes diagnosticados con DM1.

Es posible que ambas formas de diabetes afecten de formas diferentes el rendimiento cognitivo toda vez que la fisiopatología de ambas enfermedades es distinta y con edades de presentación disímiles, por lo que es razonable considerar que pudieran existir diferencias en cuanto al deterioro cognitivo asociado a cada una (39).

Fontbonne et al. (40) reportan una disminución en el desempeño de la atención, velocidad psicomotora y memoria luego de 
cuatro años de seguimiento en sujetos diagnosticados con diabetes, en edades entre los 59 a 71 ańos, en comparación con dos grupos; un grupo control y un grupo sin diagnóstico de diabetes, pero con alteraciones de los niveles de glucosa en ayunas.

Nooyens et al. (15) al medir el desempeńo cognitivo en una población de 2613 hombres y mujeres, en edades entre 40 a 73 ańos, mediante la aplicación de pruebas cognitivas dos veces en un intervalo de tiempo de 5 años, encontraron un declive dos veces mayor en memoria, flexibilidad cognitiva y funciones cognitivas globales de pacientes diagnosticados con DM2 (DM2 prevalente) respecto de los individuos que no presentaban diabetes. Con un marcado efecto de interacción entre la presentación de diabetes y la edad en la asociación entre diabetes y los posibles cambios en la flexibilidad cognitiva, motivo por el cual los autores dividen el análisis de la flexibilidad cognitiva entre pacientes en edades mayores o iguales a 60 ańos y menores de 60 años.

A su vez, Van den Berg et al. (41) al evaluar razonamiento abstracto, memoria, velocidad de procesamiento de la información, atención y funciones ejecutivas y visuoconstrucción, en 68 pacientes con DM2 y 38 controles no diabéticos en un intervalo de 4 años, describen un detrimento moderado en la velocidad de procesamiento de la información en comparación con el grupo control. Aun cuando para razonamiento abstracto y atención y funciones ejecutivas también se observa un declive, no hay dife- rencias estadísticamente significativas entre el grupo DM2 y grupo control que permitan inferir una posible relación entre la presencia de DM2 y el declive en estos dominios cognitivos. Estos autores en contraste con los datos reportados por Nooyens et al. (15) en una población similar, no describen algún declive o disminución significativa en memoria, así como tampoco describen cambios en el dominio visuoconstrucción.

Así mismo, Velayudhan et al. (42) han reportado la presencia de DM2 como un significativo predictor en el desarrollo de demencia en adultos mayores. Novik et al. (43) reportan una asociación significativa entre la presencia de DM2 y la ocurrencia de deterioro cognitivo en una población chilena con una edad promedio mayor a los 70 años. Heidari et al. (44) observaron un bajo rendimiento al evaluar la memoria en pacientes con un tiempo de diagnóstico de DM2 mayor a 5 ańos, así como en aquellos recientemente diagnosticados, sin diferencias significativas en el desempeño de la memoria entre los dos grupos. Es de importancia resaltar que en niños y adolescentes no se ha encontrado evidencia de deterioro cognitivo relacionado con la diabetes (45).

De acuerdo con Ho et al. (19) los trastornos cognitivos en pacientes diabéticos parecen estar relacionados con alteraciones en neuroplasticidad, y adicionalmente, con una reducción en la acción central de la insulina la cual regula mecanismos neurales importantes para el control de procesos de la memoria (46). Paralelamente la hiperglucemia 
crónica induce un alto estrés químico que favorece procesos celulares degenerativos especialmente en las células del hipocampo (19), estructura ligada a capacidades cognitivas como la memoria de trabajo (47). Willette et al. (48) muestran en su estudio que la resistencia a la insulina se asocia a un menor metabolismo de glucosa global a nivel cerebral, disminución que es particularmente significativa en el lóbulo temporal medial, el hipocampo, el cíngulo rostral y posterior y las regiones mediales de las cortezas parietal superior y occipital. Además, reportaron hipometabolismo bilateral en el cerebelo.

Al parecer la insulina es un modulador de los procesos cognitivos. Una de las primeras sugerencias de que la insulina podría desempeñar un papel importante en procesos cognitivos, proviene de los estudios de Zhao et al. (49) quienes demostraron que el entrenamiento en una tarea espacial aumentó la expresión del receptor de la insulina del hipocampo. La insulina hipocampal parece ser un componente clave de los procesos de memoria, y en el caso de la resistencia sistémica a la insulina que se presenta en la DM2 se ha asociado con déficit cognitivo (21). Por lo tanto, las alteraciones en la señalización de insulina cerebral pueden brindar una explicación en la ruta de los efectos cognitivos y neurales de la DM2.

La DM2 es un desorden metabólico altamente complejo y por lo tanto es de esperar que la fuente de cualquier deficiencia cognitiva asociada con ella presente un origen en el que confluyan múltiples factores (33) que interactúen a través de vías como; estrés oxidativo, glicación no enzimática de proteínas, cambios epigenéticos, inflamación crónica (17) neurodegeneración (50,51), enfermedad cerebrovascular $(52,53)$ o atrofia en regiones corticales tal como ocurre en la enfermedad de Alzheimer (50).

Tanto los productos finales de glicación, como la oxidación excesiva de moléculas de glucosa (glicooxidación) han sido reportados en concentraciones elevadas en pacientes con DM2 en comparación con no diabéticos (54-56). Se ha detectado un significativo incremento en la concentración de productos finales de glicación en pacientes con DM2 que presentan deterioro cognitivo respecto de aquellos pacientes con DM2 que no presentan deterioro cognitivo (18). Los productos finales de glicación no enzimática de proteínas, así como oxidación excesiva de moléculas de glucosa, se deben a condiciones de hiperglicemia, particularmente frecuentes en los casos de diabetes, y causan destrucción de las células al atacar y dañar sus proteínas estructurales, lo cual conlleva al deterioro de estructuras como el sistema nervioso central, particularmente frágil a estos ataques.

Adicionalmente se han observado mayores concentraciones de biomarcadores de neurodegeneración en líquido cefalorraquídeo (LCR) tales como oligómeros de Beta amiloide 1- 42 (Aß1-42), proteína tau total y proteína tau fosforilada en pacientes con trastornos cognitivos asociados a DM2, 
mecanismos que favorecen el deterioro de las habilidades cognitivas. También se ha asociada la DM2 con un menor grosor de regiones corticales como; giro y surco subcentral bilateral, surco precentral inferior derecho, gyrus rectus, surco frontal y medio (lóbulo frontal) y gyrus parietal inferior (50). Por lo tanto, es probable que la diabetes sea promotora de neurodegeneración, mecanismo que favorece el deterioro de las habilidades cognitivas. Así pues, como en una cascada de eventos desfavorables el deterioro de las habilidades cognitivas conlleva a su vez un deterioro en la calidad de vida del paciente diagnosticado con DM2 $(57,39)$

Además, Fomiga et al (58) realizaron un análisis de diversos estudios que muestran la existencia de una asociación epidemiológica entre la DM y la demencia, específicamente la han relacionado con demencia vascular y con demencia tipo Alzheimer, entre los mecanismos potencialmente implicados en la relación del deterioro cognitivo y DM2 se encuentran la microangiopatia, hiperglucemia, hipoglucemia y la resistencia a la insulina.

Así mismo, se ha propuesto el término de diabetes tipo 3 para referirse a la resistencia a la insulina, entendida esta, como una disminución de la insulina cerebral que genera una inadecuada regulación de la enzima degradante de la insulina y la acumulación de beta-amiloide. De esta manera se brinda una visión integradora de los posibles mecanismos patogenicos comunes entre la DM2 y EA (58).
Dominguez et al. (59) examinaron los estudios originales de pruebas básicas, bioquímicas y clínicas con pacientes de Alzheimer, DM2 y pacientes con Alzheimer y DM2, concluyendo que, aunque existe una relación entre ambas, esta no está claramente esclarecida. Las hipótesis de dicha relación se presentan respecto a las alteraciones en las funciones de la insulina (resistencia y señalización), lesiones microcirculatorias, cambios metabólicos que llevan a la formación de los ovillos neurofibrilares y depósitos de proteína beta-amiloide en el tejido cerebral, desequilibrio oxidante y antioxidante (vías metabólicas y mecanismo de acción diferentes pero que confluyen en el contexto de estrés oxidativo mediado a través de radicales libres). No obstante, y de acuerdo con los autores, el deterioro cognitivo parece ser menor cuando Alzheimer y DM2 se presentan de forma concomitante en comparación con aquellos pacientes que solo presentan Alzheimer.

Por su parte, las lesiones de la materia blanca, en especial en adultos mayores, han sido asociadas con deficiencias cognitivas (60) inteligencia, atención y funciones ejecutivas, sin embargo, no se ha establecido una clara relación entre la diabetes como factor de riesgo y la ocurrencia de lesiones de la materia blanca (61). Aun cuando la resistencia a la insulina ha demostrado una fuerte correlación con este tipo de lesiones (62, 63), tambien las fluctuaciones en los niveles de glucosa determinados a través de hemoglobina glicosilada (HbA1c), parecen 
jugar un importante papel en el declive cognitivo de pacientes con DM2 (64).

De acuerdo con Li y Huang (22) los mecanismos implicados en el deterioro cognitivo de pacientes con DM2, así como los mecanismos implicados en el desarrollo de demencia en pacientes con DM2 presentan una ruta común, en la cual la neurodegeneración es consecuencia de las alteraciones metabólicas en los ciclos de glúcidos, envejecimiento, hipertensión, el padecimiento de DM2 por un largo tiempo y elevados niveles de colesterol.

Además, la predisposición genética contribuye al aumento de riesgo de demencia en presencia de DM2. El alelo APOE e4 es el factor genético más examinado en el aumento del riesgo de demencia asociada a DM (58).

Así como los anteriores estudios muestran que la diabetes está asociada al deterioro cognitivo, existen otros estudios como los de Colberg et al. (65) que evidencian que la actividad física reduce este riesgo. Blondell et al. (66) realizaron una revisión de estudios sobre la asociación de la actividad física y deterioro cognitivo y actividad física y demencia; el metanálisis sugiere que mayores niveles de actividad física en comparación con niveles más bajos tienen un riesgo reducido de deterioro cognitivo y demencia. Finalmente, una de las más recientes revisiones de literatura sobre este tema fue realizada por Hernández et al. (67) en la cual señalan que la actividad física en personas con DM2 disminuye el riesgo cardiovascular ya que mejora el control metabólico, el peso y la sensibilidad a la insulina; por lo que la actividad física es señalada como uno de los pilares del tratamiento integral para la DM2.

\section{Conclusiones}

La DM2 es una enfermedad cronica, con complicaciones asociadas, que implica un alto costo economico y social con altas repercusiones en la salud publica. Aun cuando se han generado numerosos estudios que han mostrado una posible relación entre la deficiencia cognitiva y la DM2, en la actualidad no se ha logrado un consenso que establezca el nivel de asociación entre estas dos variables o el grado de afectación de dominios cognitivos.

Diversos estudios reportan alteración en algunos de los dominios cognitivos, en la atención, la memoria de trabajo, la memoria verbal, memoria visual, el aprendizaje y la función ejecutiva $(24,40,15,41)$, indispensables en el procesamiento mental de la información y los cuales resultan de relevancia para el adecuado desenvolvimiento social del paciente y la adhesión de los tratamientos que se fundamentan en el auto cuidado.

Otros estudios indican una asociación significativa entre la presencia de DM2 (43) y la ocurrencia de deterioro cognitivo y también 
como un predictor en el desarrollo de demencia en adultos (42) y demencia tipo Alzheimer

La asociación entre el deterioro cognitivo en pacientes con DM2 se han relacionado con alteraciones en la neuroplasticidad (46), resistencia y señalización de la insulina (48), lesiones en la materia blanca (60), estrés oxidativo, glicación no enzimática de proteínas, cambios epigenéticos, inflamación crónica (17) neurodegeneración (50,51), enfermedad cerebrovascular $(52,53)$ o atrofia en regiones corticales tal como ocurre en la enfermedad de Alzheimer, al observar mayor concentraciones de biomarcadores de neurodegeneración en líquido cefalorraquídeo (LCR) tales como oligómeros de Beta amiloide 1- 42 (Aß1-42), proteína tau total y proteína tau fosforilada (50) y dentro de los factores determinantes del grado de deterioro cognitivo se describe la duración de la DM2 y la presencia de complicaciones por coomorbilidades, enfermedad metabólica e hipertensión arterial.

Mientras, la asociación observada entre riesgo vascular y la resitencia a la insulina, a menudo denominada síndrome metabólico (SM), ha llevado a pensar que el riesgo cardiovascular aparece precozmente, antes de que se desarrolle la DM2, mientras que la relación estrecha entre hiperglucemia y enfermedad microvascular (encefalopatía, retinopatía, nefropatía, neuropatía) indica que este riesgo no se vuelve aparente hasta que aparece una hiperglucemia franca. Estos conceptos ponen de manifiesto la natu- raleza progresiva tanto de la DM2 como del riesgo vascular asociado, lo que comporta desafíos específicos en las distintas fases de la vida de una persona con DM2. Los efectos de la edad avanzada, las comorbilidades y los problemas asociados con cada grupo específico indican la necesidad de enfocar el riesgo tomando en cuenta las características biopsicosociales e individualizantes que permitan brindar al paciente herramientas integrales para el manejo de su propia enfermedad.

Finalmente, para poder responder de forma contundente si el deterioro cognitivo es una complicación de la DM2 se concluye la necesidad de desarrollar investestigaciones longitudinales que incluyan diferentes tipos de variables, como son: el tiempo de diagnostico de la enfermedad, la adherencia al tratamiento, las comorbilidades, el alelo APOE e4, resonancia magnetica, hemoglobina glicosilada, y la evaluación neurocognitiva y afectiva.

A la vez, al revisar las diferentes investigaciones es considerable realizar prevención del deterioro cognitivo en pacientes diagnosticados con DM2, a partir de una evaluación integral que incluya pruebas neuropsicológicas dentro de los exámenes de rutina, junto a un acompańamiento integral, en pro de mejorar el estilo y la calidad de vida del paciente, mediante un control de la comorbilidad de las enfermedades asociadas a la DM2.Además del manejo de emociones y el estrés y en mayor medida en pacientes con largo tiempo de diagnóstico de la enferme- 
dad y en aquellos donde se identifique que un déficit cognitivo que pueda estar condicionando la adherencia al tratamiento.

\section{De igual forma, se sugiere incluir dentro de las intervenciones integrales la realización de actividad física, el mantenimiento de una alimentación adecuada, y niveles con- trolados de la hemoglobina glicosilada.}

\section{Referencias}

1. Federación Internacional de la Diabetes. Atlas de la Diabetes de la FDI, novena edición. Bruselas, Bélgica; 2019. Disponible en: https://www.diabetesatlas.org

2. Ogurtsova K; da Rocha-Fernandes J; Huang Y; Linnenkamp U; Guariguata L; Cho N; et al. IDF Diabetes Atlas: Global estimates for the prevalence of diabetes for 2015 and 2040. Diabetes Res Clin Pract. 2017 Jun; 128:40-50. https://www.diabetesresearchclinicalpractice.com/article/S01688227(17)30375-3/fulltext

3. Organización Mundial de la Salud - OMS. Informe mundial sobre la diabetes. Ginebra, Suiza; 2016. Disponibel en: https://apps.who.int/iris/bitstream/ handle/10665/254649/9789243565255-spa.pdf;jsessionid=3D676CEB4FC964428007FCD1B1EFD8E8? sequence $=1$

4. Federación Internacional de la Diabetes - IDF. Atlas de la diabetes de la FDI, séptima edición. Bruselas, Bélgica; 2015. Disponible en: https://www.idf. org/e-library/epidemiology-research/diabetes-atlas

5. Ministerio de Salud y Protección Social, y Departamento Administrativo de Ciencia, Tecnología e Innovación - Colciencias. Guía de práctica clínica para el diagnóstico, tratamiento y seguimiento de la diabetes mellitus tipo 2 en la población mayor de 18 ańos, Guía No. GPC-2015-51. Bogotá; 2016. Disponible en: http://gpc.minsalud.gov.co/ gpc_sites/Repositorio/Conv_637/GPC_diabetes/ Guia_Diabetes_Profesionales_Tipo_2.pdf
6. Murray RK, Bender DA, Botham KM, Kennelly PJ, Rodwell VW, Weil PA. Harper Bioquímica ilustrada. 28a edición. México: McGRAW-Hill Interamericana Editores, S.A; 2010. pp. 131-142.

7. American Diabetes Association - ADA. Diagnosis and Classification of Diabetes Mellitus. Diabetes Care. 2011; 34(1):S62-S69. Disponible en: https://care.diabetesjournals.org/content/34/Supplement_1/

8. Asociación Panamericana de Oftalmología APAO; Programa Visión 2020/IAPB Latinoamérica; Consejo Internacional de Oftalmología - ICO. Actualización de la Guía clínica de Retinopatía Diabética para Latinoamérica. Buenos Aires; 2016. Disponible en: https://oftalmologos.org.ar/ noticias/620-actualizacion-de-la-guia-de-retinopatia-diabetica.

9. Campuzano-Maya MG; Latorre-Sierra G. La HbA1c en el diagnóstico y en el manejo de la diabetes. Medicina \& Laboratorio, 2010; 16:211-241. https://biblat.unam.mx/hevila/Medicinalaboratorio/2010/vol16/no5-6/1.

10. Miles WR; Root HF. Psychologic tests applied to diabetic patients. Arch Intern Med, 1992; 30(6):767-777. https://jamanetwork.com/journals/jamainternalmedicine/article-abstract/534090

11. Dejgaard A; Gade A; Larsson H; Balle V; Parving A; Parving H. Evidence for diabetic encephalopathy. Diabet Med. 1991; 8(2):162-167. https://onlinelibrary.wiley.com/toc/14645491/1991/8/2

12. Gaudieri PA; Chen R; Greer TF; Holmes CS. Cognitive function in children with type 1 diabetes: a meta-analysis. Diabetes care. 2008; 31(9):18921897. https://care.diabetesjournals.org/content/31/9/1892.short\#aff-1

13. Lu FP; Lin KP; Kuo HK. Diabetes and the risk of multi-system aging phenotypes: a systematic review and meta-analysis. PLoS One. 2009; 4(1), e4144. https://journals.plos.org/plosone/article?id=10.1371/journal.pone. 0004144

14. Cheng G; Huang C; Deng H; Wang H. Diabetes as a risk factor for dementia and mild cognitive impairment: a meta-analysis of longitudinal studies. Intern Med J. 2012; 42(5):484-91. https://onlinelibrary.wiley.com/toc/14455994/2012/42/5 
15. Nooyens A; Baan CA; Spijkerman A; Verschuren WM. Type 2 diabetes and cognitive decline in middle-aged men and women: the Doetinchem Cohort Study. Diabetes Care. 2010; 33(9):1964-9. https://care.diabetesjournals.org/content/33/9/1964

16. Stratton IM; Adler AI; Neil HAW; Mattews DR; Manley SE; Cull CA; et al. Association of glucaemia with macrovascular and microvascular complications of type 2 diabetes (UKPDS 35): Propspective observational study. BMJ. 2000; 321:405-12. https://www.bmj.com/content/321/7258/405

17. Testa R; Bonfigli AR; Prattichizzo F; La Sala L; De Nigris V; Ceriello A. The "Metabolic Memory" Theory and the Early Treatment of Hyperglycemia in Prevention of Diabetic Complications. Nutrients. 2017; 9(5):437. https://www.mdpi. com/2072-6643/9/5/437

18. Gorska-Ciebiada M; Saryusz-Wolska M; Borkowska A; Ciebiada M; Loba J. (2015). C-Reactive Protein, Advanced Glycation End Products, and Their Receptor in Type 2 Diabetic, Elderly Patients with Mild Cognitive Impairment. Front. Aging Neurosci. 2015; 7:209. https://www.frontiersin.org/articles/10.3389/fnagi.2015.00209/full

19. Ho N; Sommers MS; Lucki I. Effects of Diabetes on Hippocampal Neurogenesis: Links to Cognition and Depression. Neurosci Biobehav Rev. 2013; 37(8): 1346-1362. https://www.sciencedirect. com/science/article/abs/pii/S0149763413000705

20. McEwen BS. Protective and damaging effects of stress mediators: central role of the brain. Dialogues Clin Neurosci. 2006; 8(4):367-381 https:// www.dialogues-cns.org/contents-8-4/

21. McNay EC; Recknagel AK. Brain insulin signaling. A key componet of cognitive processes and a potential basis for cognitive impairment in type 2 diabetes. Neurobiol Learn Mem. 2011; 96(3):432442. https://www.sciencedirect.com/science/article/abs/pii/S1074742711001493

22. Li W; Huang E. An update on type 2 diabetes mellitus as a risk factor for dementia. J. Alzheimers. Dis. 2016; 53(2): 393-402. https://www.j-alz.com/ vol53-2
23. Brands AM; Biessels GJ; Kappelle LJ; de Haan EH; de Valk HW; Algra A; et al. Cognitive functioning and brain MRI in patients with type 1 and type 2 diabetes mellitus: a comparative study. Dement Geriatr Cogn Disord. 2007; 23(5):343-350. https://www.karger.com/Article/Abstract/100980

24. Reijmer YD; Brundel M; de Bresser J; Kappele LJ; Leemans A; Biessels GJ. (2013). Microstructural White Matter Abnormalities and Cognitive Funcioning in Type 2 Diabetes. Diabetes Care. 2013; 36(1):137-144. https://care.diabetesjournals.org/ content/36/1/137

25. Strachan MWJ; Deary IJ; Ewing FME; Frier $\mathrm{BM}$. Is type II diabetes associated with an increased risk of cognitive dysfunction? A critical review of published studies. Diabetes Care. 1997; 20(3):438-445. https://care.diabetesjournals.org/ content $/ 20 / 3 / 438$

26. American Diabetes Association - ADA. Standards of medical care in diabetes-2017: Summary of Revisións. Diabetes Care. 2017; 40(1):S4-S5. https://care.diabetesjournals.org/content/40/Supplement_1/S4

27. Federación Internacional de la Diabetes - IDF. IDF Clinical Practice Recommendations for managing Type 2 Diabetes in Primary Care. Bruselas, Belgica; 2017. Disponible en: https://idf.org/our-activities/ advocacy-awareness/resources-and-tools/128:i$\mathrm{df}$-clinical-practice-recommendations-for-managing-type-2-diabetes-in-primary-care.html

28. Inzucchi SE; Bergenstal RM; Buse JB; Diamant M; Ferrannini E; Nauck M; et al. Management of hyperglycaemia in type 2 diabetes, 2015: a patient-centred approach. Update to a Position Statement of the American Diabetes Association and the European Association for the Study of Diabetes. Diabetes Care. 2015; 38(1):140-149. https:// care.diabetesjournals.org/content/38/1/140

29. Sternberg RJ; Sternberg K. Cognitive Spychology, Sixth edition. Belmont, California.: Wadsworth, Cengage Learning.; 2012.

30. Neisser U. Cognitive psychology. Englewood Cliffs, USA: Prentice-Hall.; 1967. 
31. Hyman, I. The ralling cry for the cognitive revolution. En: Neisser U. Cognitive Psychology: Classic Edition. New York: Psychology Press; 2014. p. XVXIX.

32. Neisser U. Cognitive psychology: Classic Edition. New York, USA.: Psychology Press.; 2014.

33. Strachan MWJ. R D Lawrence Lecture 2010. The brain as a target organ in Type 2 diabetes: exploring the links with cognitive impairment and dementia. Diabet Med. 2011; 28(2):141-147. https:// onlinelibrary.wiley.com/doi/full/10.1111/j.14645491.2010.03199.x

34. Ryan CM; Geckle MO; Orchard TJ; Cognitive efficiency declines over time in adults with Type 1 diabetes: effects of micro- and macrovascular complications. Diabetologia. 2003; 46(7):940-948. https://link.springer.com/article/10.1007/s00125003-1128-2

35. Brands AMA; Biessels GJ; de Haan EH; Kappelle LJ; Kessels RP. The effects of type 1 diabetes on cognitive performance: a meta-analysis. Diabetes Care. 2005;28(3):726-735. https://care.diabetesjournals.org/content/28/3/726.long

36. Kodl CT; Seaquist ER. Cognitive dysfunction and diabetes mellitus. Endocr Rev. 2008; 29(4):494-511. https://academic.oup.com/edrv/ article/29/4/494/2355063

37. Wrighten SA; Piroli GG; Grillo CA; Reagan LP. A look inside the diabetic brain: Contributors to diabetes-induced brain aging. Biochim Biophys Acta. 2009; 1792(5):444-453. https://www.sciencedirect.com/science/article/pii/ S092544390800210X?via\%3Dihub

38. Jacobson AM; Musen G; Ryan CM; Silvers N; Cleary P; Waberski B. Long-term effect of diabetes and its treatment on cognitive function. $\mathrm{N}$ Engl J Med. 2007; 356(18):1842-52. https://www.nejm. org/doi/full/10.1056/nejmoa066397

39. Díaz-Venegas C; Schneider DC; Myrskylä M; Mehta NK. Life expectancy with and without cognitive impairment by diabetes status among older Americans. PLoS One [Internet]. 2017 [consultado 30 de marzo de 2018]; 12(12). Disponible en: https:// journals. plos.org/plosone/article?id=10.1371/journal.pone. 0190488
40. Fontbonne A; Berr C; Ducimetière P; Alpérovitch, A. Changes in cognitive abilities over a 4-year period are unfavorably affected in elderly diabetic subjects: results of the Epidemiology of Vascular Aging Study. Diabetes Care. 2001; 24(2):366-370. https://care.diabetesjournals.org/content/24/2/366

41. Van den Berg E; Reijmer YD; de Bresser J; Kessels RP; Kappelle LJ; Biessels GJ; et al. A 4 year follow-up study of cognitive functioning in patients with type 2 diabetes mellitus. Diabetología. 2010; 53(1):58-65. https://link.springer.com/article/10.1007/s00125-009-1571-9

42. Velayudhan L; Poppe M; Archer N; Proitsi P; Brown RG; Lovestone S. Risk of developing dementia in people with diabetes and mild cognitive impairment. Br J Psychiatry. 2010; 196(1):36-40. https://www.cambridge.org/core/ journals/the-british-journal-of-psychiatry/article/ risk-of-developing-dementia-in-people-with-diabetes-and-mild-cognitive-impairment/66 FC681F46B03CBC17AAAE0A6B4E030B

43. Novik V; García S; Heller F; Mercado J. Influencia de la Diabetes Mellitus tipo 2 en el deterioro cognitivo en un grupo de adultos mayores. Rev. chil. endocrinol. diabetes. 2011; 4 (1):13-17. http://www. revistasoched.cl/1_2011/index-1-2011.html

44. Heidari M; Ghahremanlu H; Haghshenas M; Sadeghi M; Heidari A. Comparison of memory impairments among two groups of patients with diabetes with different disease durations. BMC Research Notes. 2012; 5:353. http://www.biomedcentral. $\mathrm{com} / 1756-0500 / 5 / 353$

45. Pourabbasi A; Tehrani-Doost M; Qavam SE; Farzami J; Larijani B. Evaluation of Cognitive Functions in Iranian Children and Adolescents with Diabetes Mellitus. Acta Med Iran. 2017; 55(6):381-388. https://acta.tums.ac.ir/index.php/acta/article/ view/6197

46. Kern W; Peters A; Fruehwald-Schultes B; Deninger E; Fehm HL. Improving influence of insulin on cognitive dunctions in humans. Neuroendocrinology. 2001; 74(4):270-280. https://www.karger. com/Article/Abstract/54694 
47. Rudner M; Fransson P; Ingvar M; Nyberg L; Rönnberg J. Neural representation of binding lexical signs and words in the episodic buffer of working memory. Neuropsychologia, 2007; 45(10):22582276. https://www.sciencedirect.com/science/article/abs/pii/S0028393207000772?via\%3Dihub

48. Willette AA; Bendlin BB; Starks EJ; Birdsill AC; Johnson SC; Christian BT. Association of Insulin Resistance with Cerebral Glucose Uptake in Late Middle-Aged Adults at Risk for Alzheimer Disease. JAMA Neurol. 2015; 72(9):1013-1020. https:// jamanetwork.com/journals/jamaneurology/fullarticle/2398420

49. Zhao W; Chen H; Xu H; Moore E; Meiri N; Quon $\mathrm{MJ}$; et al. Brain insulin receptors and spatial memory. Correlated changes in gene expression, tyrosine phosphorylation, and signaling molecules in the hippocampus of water maze trained rats. J Biol Chem. 1999; 274(49). https://www.jbc.org/content/274/49/34893.long

50. Moran C; Beare R; Phan TG; Bruce DG; Callisaya ML; Srikanth V. Type 2 diabetes mellitus and biomarkers of neurodegeneration. Neurology. 2015; 85(13):1123-30. https://n.neurology.org/content/85/13/1123.long

51. Moran C; Phan TG; Chen J; Blizzard L; Beare R; Venn A; Srikanth V. Brain atrophy in Type 2 diabetes: regional distribution and influence on cognition. Diabetes Care. 2013; 36(12):40364042. https://care.diabetesjournals.org/content/36/12/4036

52. Qiu C; Sigurdsson S; Zhang Q; Jonsdottir M K; Kjartansson O; Eiriksdottir G; Launer LJ. Diabetes, markers of brain pathology and cognitive function: the age, gene/environment susceptibility-Reykjavik study. Ann Neurol. 2014; 75(1):138146. https://www.ncbi.nlm.nih.gov/pmc/articles/ PMC4540233/

53. Callisaya ML; Daly RM; Sharman JE; Bruce D; Davis TME; Greenaway T; et al. Feasibility of a multi-modal exercise program on cognition in older adults with Type 2 diabetes - a pilot randomised controlled trial. BMC Geriatr. 2017; 17(1). https://bmcgeriatr.biomedcentral.com/articles/10.1186/s12877-017-0635-9
54. Kalousová M; Skrha J; Zima T. Advanced glycation end-products and advanced oxidation protein products in patients with diabetes mellitus. Physiol Res, 2002; 51(6):597-604. https://pubmed.ncbi. nlm.nih.gov/12511184/

55. Krapfenbauer K; Birnbacher R; Vierhapper H; Herkner K; Kampel D; Lubec G. Glycoxidation, and protein and DNA oxidation in patients with diabetes mellitus. Clin Sci (Lond). 1998; 95(3):331337. https://pubmed.ncbi.nlm.nih.gov/9730853/

56. Nowotny K; Jung T; Höhn A; Weber D; Grune T. Advanced glycation end products and oxidative stress in type 2 diabetes mellitus. Biomolecules. 2015;5(1):194-222. https://www.mdpi.com/2218$273 X / 5 / 1 / 194$

57. Sinclair AJ; Girling A J; Bayer A. Cognitive dysfunction in older subjects with diabetes mellitus: impact on diabetes selfmanagement and use of care services. Diabetes Res Clin Pract, 2000;50(3):203212. https://www.diabetesresearchclinicalpractice. com/article/S0168-8227(00)00195-9/fulltext

58. Formiga F; Reñe R; Pérez M. Dementia and diabetes: Casual or Causal Relationship?. Rev. Med. Clin. (Barc), 2015;144(4):176-80. https://www.elsevier.es/es-revista-medicina-clinica-2-linkresolver-demencia-diabetes-relacion-casual-o-S0025775314001195

59. Dominguez RO; Pagano MA; Marschoff ER; González SE; Repetto MG; Serra JA. Enfermedad de Alzheimer y detorio cognitivo asociado a la diabetes mellitus tipo 2: relaciones e hipótesis. Revista de Neurología. 2014; 29(9):567-572. https://www. elsevier.es/es-revista-neurologia-295-articulo-enfermedad-alzheimer-deterioro-cognitivo-asociado-S0213485313001552

60. Roriz-Filho SJ; Sa-Roriz TM; Rosset I; Camozzato AL; Santos AC; Chaves ML; et al. (Pre)diabetes, brain aging, and cognition. Biochim Biophys Acta. 2009; 1792(5):432-43. https://www.sciencedirect. $\mathrm{com} /$ science/article/pii/S0925443908002524?via\%3Dihub

61. You CJ; Liu D; Liu LL; Li GZ. Correlation between acute stroke-induced white matter lesions and insulin resistance. Medicine (Baltimore). 2018; 97(11):e9860. https://www.ncbi.nlm.nih. gov/pmc/articles/PMC5882385/ 
62. Rundek T; Gardener H; Xu Q; Goldberg RB; Wright $\mathrm{CB}$; Boden-Albala $\mathrm{B}$; et al. Insulin resistance and risk of ischemic stroke among nondiabetic individuals from the northern Manhattan study. Arch Neurol. 2010; 67(10):1195-200. https://jamanetwork.com/journals/jamaneurology/fullarticle/801407

63. Anan F; Masaki T; Kikuchi H; Iwao T; Shimomura T; Umeno Y; et al. Association between plasma highsensitivity $\mathrm{C}$-reactive protein and insulin resistance and white matter lesion in Japanese type 2 diabetic patients. Diabetes Res Clin Pract. 2010; 87(2):233-239. https://www.diabetesresearchclinicalpractice.com/article/S0168-8227(09)004653/fulltext

64. Feinkohl I; Aung PP; Keller M; Robertson CM; Morling JR; McLachlan S; et al. Severe hypoglycemia and cognitive decline in older people with type 2 diabetes: The Edinburgh type 2 diabetes study. Diabetes Care. 2014; 37(2):507-515. https://care. diabetesjournals.org/content/37/2/507.long

65. Colberg SR; Somma CT; Sechrist SR. Physical Activity Participation May Offset Some of the Negative Impact of Diabetes on Cognitive Function. J. Am. Med. Dir. Assoc. 2008; 9(6):434-438. https:// www.jamda.com/article/S1525-8610(08)00121-7/ fulltext

66. Blondell SJ; Hammerseley-Mather R; Veerman JL. Does physical activity prevent cognitive decline and dementia A sistematic review and meta-analysis of longitudinal studies. BMC Public Health. 2014; 14(510). https://bmcpublichealth.biomedcentral.com/articles/10.1186/1471-2458-14-510

67. Hernández RJ; Domínguez YA; Mendoza-Choqueticlla J. Efectos benéficos del ejercicio físico en las personas con diabetes mellitus tipo 2. Rev. Cuba. Endoc. 2018; 29(2):1-18. https://www.medigraphic.com/cgi-bin/new/resumen.cgi?IDARTICU$\mathrm{LO}=84725$ 